\title{
PILEP: a contribution to PCE-based interdomain path computation
}

\author{
M. Domínguez-Dorado, José-Luis González-Sánchez, J. Domingo-Pascual
}

\begin{abstract}
The process of computing routes that network traffic must follow throughout Internet has become more complex in the last years. Nowadays, this process is subject to the application of several constraints related to traffic engineering, resources management, quality of the offered services, security or robustness. The application of all these constraints has caused an increase of complexity of those nodes in charge of path computation. Sometimes, the amount of time and resources spent to compute routes is superior to resources used in the main task of these nodes: traffic classification and forwarding. PCE (Path Computation Element) architecture is being developed to diminish that problem in the context of constraint-based path computation for MPLS (Multiprotocol Label Switching). Although research work in relation to this topic is making progress quickly, there are still some unsolved aspects. As a contribution to PCE development, in this work we present a mechanism called PILEP (Procedure for Interdomain Location of External PCEs) that allows the dynamic discovery of routes computation elements in interdomain environments, making use of the existing routing protocols.
\end{abstract}

Index terms - Inter-AS PCE discovery, MPLS, BGP, OSPF-TE, constraints, QoR.

\section{INTRODUCTION}

Since the first implementation of RIP (Routing Information Protocol), the routing mechanisms have advanced remarkably. At present, Internet integrates several heterogeneous networks where multiple technologies coexist: IP (Internet Protocol), Ethernet, ATM (Asynchronous Transfer Mode), MPLS, and so on. Each one of these technologies has its own features that affect, to a greater or lesser extent, the capacity of the network to provide a service of quality to the final users. Traffic engineering [1] and QoR [2] (Quality of Routing) are two disciplines intended to increase that capacity: they modify the Internet nature by making connection-oriented those networks

M. Domínguez-Dorado belongs to DISIT at University of Extremadura. Avda. de la Universidad s/n. CP: 10071. Cáceres, SPAIN. Phone: +34927 257 431. Fax: +34 927257 202. e-mail: mdomdor@unex.es.

José-Luis González-Sánchez belongs to DISIT at University of Extremadura. Avda. de la Universidad s/n. CP: 10071. Cáceres, SPAIN. Phone: +34 927257 431. Fax: +34 927257 202. e-mail: jlgs@unex.es.

J. Domingo-Pascual belongs to DAC at Technical University of Catalunya. Jordi Girona, Campus Norte. Módulo D6. 08034 Barcelona. SPAIN. Phone: +34 934016981. Fax: +34 934017055. e-mail: jordi.domingo@ac.upc.es. that do not have this feature; reserving resources; computing backup paths, providing fault-tolerance, etc. MPLS [3] makes easier this work by means of the integration of different technologies and their respective control planes. The confluence of these new approaches has had repercussions in the path computation process. Some decades ago, the primary target was that the traffic arrived at its destination (whenever and however). Nowadays, the paths computation mechanisms have to keep in mind new aspects never taken into account before; for example, policies application [4], economic cost minimization, network resources maximization, management of the circulating network traffic, or computation of disjoint path [5], among others.

This new situation has affected the route computation process that is now much more complex and needs more resources. Nodes in charge of traffic classification and forwarding (generally those that are entry points to the MPLS network) are those that habitually have carried out path computation tasks; however, now it is a too much hard work. Some years ago, the IETF (Internet Engineering Task Force) started to research into a new technology expected to free MPLS nodes of computing LSP (Label Switched Path) tasks, so they could dedicate all the time to LSP establishment and traffic forwarding; and the result of this research works was the definition of PCE (Path Computation Element) architecture [6].

The general purpose of our work is to contribute to the development of PCE architecture in those environments, like interdomain, where there are still unsolved topics; thus, we supply PILEP, a dynamic mechanism to locate PCEs in interAS environments.

The rest of this document is organized as follows: the second section is a succinct description of the PCE architecture. In the third section, we highlight the motivations to design and develop an interdomain PCE discovery mechanism. In the fourth section, we present PILEP our proposal for dynamic interdomain PCE discovery. In the fifth section, we carry out an explanatory example of PILEP operation. Finally, in the sixth section, conclusions and future work derived from ours are explained.

\section{DESCRIPTION OF PCE ARQUITECTURE}

PCE architecture is being developed now so, most of the RFC published by the IETF are definitions and general requirements of the architecture. The most basic PCE architecture must have at least tree key elements (Fig. 1). PCE is the element in charge of paths computation. PCC (Path 


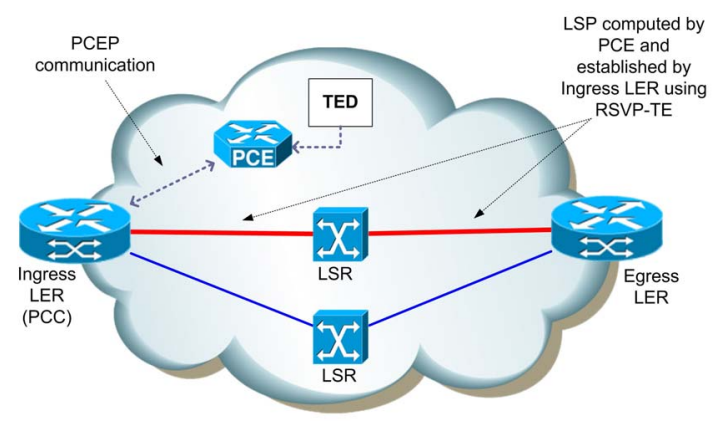

Fig. 1. Key components of PCE architecture.

Computation Client) is the client that will request the PCE for a path computation; and PCEP (Path Computation Element communication Protocol) [7], [8], is the communication protocol throughwhich PCEs and PCCs are communicated. In general, LER (Label Edge Router) will act as PCC because they are access points to MPLS network.

Even though at first glance it might appear to be a very simple model, some difficulties come up when integrating PCE in an autonomous system based on legacy technologies. Researchers around the world are managing some of them, e.g. the relationship of PCE and IGPs (Interior Gateway Protocol) and EGPs (Exterior Gateway Protocol), the way that existing protocols feeds PCE with traffic engineering information, and so on.

In PCE architecture, a given AS (Autonomous System) or MPLS domain must have one or more PCE elements in charge of computing LSPs inside it. Each node that wants to start an LSP establishment will have to proceed as a PCC; consequently, at least LER nodes must act as PCC because they have the responsibility of establishing LSPs in the interior of the autonomous system. Besides them, other intermediate nodes could need to act as PCC if they are currently involved in local path restoration mechanisms and need to compute routes.

As one flow arrives to an ingress LER, that LER will act as a PCC and ask PCE for a path computation from itself to the egress LER. In order to do it, it will use PCEP protocol, which provides enough functionality to allow it to make the request in a feasible way. The request will incorporate a set of constraints the PCC must take into account when computing the path. PCE will calculate the route based on the information included in its TED (Traffic Engineering Database), a database that contains the link-state graph and any other information that could be of helpfulness. Each PCE is associated to a TED that is periodically updated by IGPs or by any other method that could be defined. Once that the PCE has computed the requested path, it will use PCEP yet again to send back a response to the ingress LER/PCC that sent the initial request.

In the requirements description of PCEP [7], it is specified that a computed path, included in the reply to a LER/PCC, must be directly mapped into an RSVP-TE (Resource Reservation Protocol - Traffic Engineering) ERO [9] (Explicit Routing Object) object so, the LER/PCC is able to start the LSP establishment using RSVP-TE and that ERO object.
This is the most basic operation mode of the PCE architecture (simple path computation). PCE allows the existence of other more complex situation to coordinate and set up, as we can see in Fig. 2. For example, more than one PCE element can be allocated inside an AS, each one of them in charge of computing complete LSPs. In that case, a given LER/PCC will have the opportunity of choosing, among them, the PCE that fits better to its necessities. That situation obliges the LER/PCC to be aware of the capabilities of each PCE in order to attain a selection process based on a reasonable criterion. Another example is the one where there are more than a single PCE inside an AS and each one of them has only the capability of computing path segments related to a specific area of the network (multiple path computation). In that case, PCEs will have the obligation to cooperate to compute each own segment, assemble them and give the initiating LER/PCC the path it asked for.

For that reason, there are some situations where a PCC has to act as PCC in face of other PCEs. Nonetheless, the most complex situation happens when the last two coincide all at once and the path requested by the LER/PCC exceeds the local AS boundary. In the latter case, PCE architecture should have mechanisms to overcome the traditional interdomain routing problems [10], [11] in relation to traffic engineering and MPLS: partial information about the topology, lack of information about traffic engineering, policy-based routing, oneness of routes, security, network recovery, resilience or disclosures of AS infrastructure.

\section{ARGUMENTS FOR DESIGNING AN INTER-AS PCE ELEMENTS DISCOVERY MECHANISM}

Regardless the case, the first step should be for PCCs to discover the existing PCE. In the simplest case, a LER/PCC will need to know the PCE that can help it to compute a route. In the case of some PCE that have to cooperate, each PCE needs to known the existence of the others, in order to make feasible this collaboration.

Interdomain LSP computation [12], [13] needs the cooperation between two or more PCE, so, in that case, the dynamic PCE discovery mechanism becomes relevant.

Next subsections will describe the existing intra-AS PCE discovery mechanisms and the difficulties of extrapolate them to interdomain environments.

\section{A. IGP-based intra-AS discovery}

In [14], it is specified that a PCC must know about the existence of a given PCE by way of any method except for broadcast. Moreover, it imposes other requirements such as the fact that a PCE can be discovered in different detail or degree by PCCs, or that the AS confidentiality needs to be preserved in the case of an interdomain discovery mechanism. According to these requirements, there are two proposals that use the OSPF (Open Shortest Path First) [19] and IS-IS (Intermediate System to Intermediate System) [20] flooding mechanism to spread the existence of PCEs within an autonomous system. It allows the interested PCCs to find out the advertised PCEs. In [19] it is defined a new OSPF TLV 


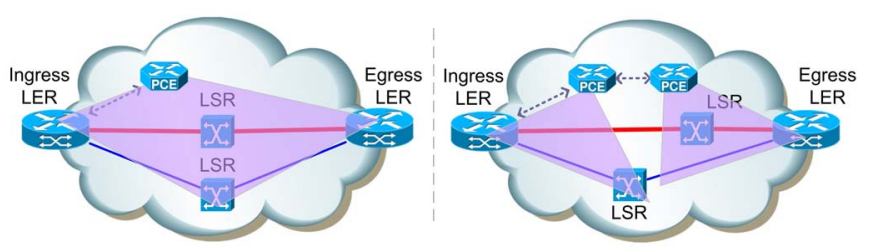

Fig. 2. Simple computation (left) and multiple computation (right).

(Type Length Value) tern called PCED (PCE Discovery) that can be inserted inside an OSPF RI-LSA (Routing Information - Link State Advertisement) [21] when using OSPF traffic engineering extensions [22]. OSPF nodes exchange RI-LSA messages so often to preserve their topological databases updated. These databases, each one owned by an OSPF node, are used to compute the next hop to the destination for the traffic.

This discovery mechanism adds to that messages additional information (PCED TLV) so that the information about the existence of a given PCE is flooded at the same time that the information about link state. In order to use that mechanism, the PCE that wants to be discovered will have to take part in OSPF operation. PCED TLV (Fig. 3) is composed of a set of unsorted sub-TLVs. These sub-TLVs contain enough information to make feasible for every PCCs and PCEs within an AS to not only discover the existing PCEs, but also to choose between them the most adequate in each moment. PCED will always carry information about the IP address that a PCC must use to reach the advertised PCE (PCE-ADDRESS sub-TLV) and information to indicate the PCE working scope (PCE-SCOPE sub-TLV), e.g. inter-AS, inter-area, inter-layer, etc.

In addition, PCED TLV may include optional information that provides a more detailed view of the advertised PCE. PCE-DOMAIN and PCE-NEIG-DOMAIN inform the PCC about the specific areas or autonomous systems on which the PCE has the capability of compute LSP. PCE-CAP-FLAGS sub-TLV includes information about specific features or capabilities of the advertised PCE that are useful to choose between some available PCEs (requests prioritization, bidirectional LSP computation capability, and so on).

At last, a PCE uses CONGESTION to indicate whether it is congested or not. Using that mechanism, a PCE that is taking part in OSPF is able to advertise itself to those PCEs or PCCs susceptible to make use of it, using the traditional IGP's

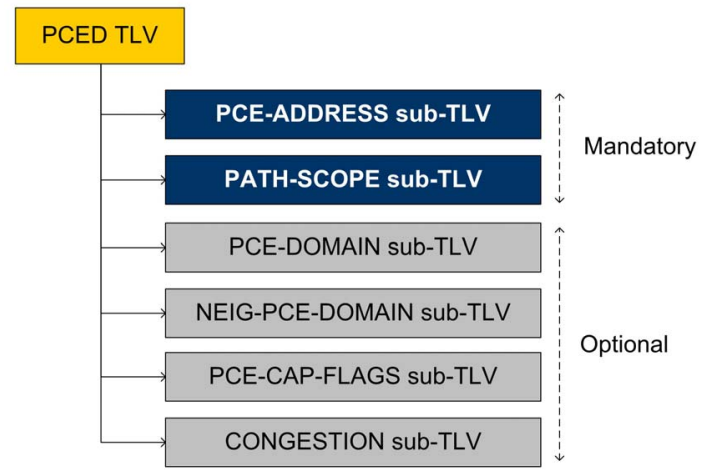

Fig. 3. TLV PCED format. flooding mechanisms. Each PCC or PCE in the scope of OSPF-TE flooding procedure will discover, avoiding the use of broadcast, the existence of a PCE as well as enough information to perform an adequate selection process (Fig. 4). This mechanism is especially positive since it takes advantage of the pre-existing techniques to spread the existence and the state of PCCs inside an AS, with minimum modifications of the existing protocols.

\section{B. Lack of inter-AS discovery mechanism}

Notwithstanding its goodness, we cannot extrapolate the aforementioned processes to an interdomain environment. IGPs supporting it have a limited scope restricted to the interior of an autonomous system and, therefore, they are not able to carry information about PCEs between adjacent ASes.

Although in [14] the requirements for a inter-AS PCE discovery method are detailed, a dynamic and automatic method has not been developed yet to allow a PCE from an autonomous system to know about the existence of PCEs in an adjacent autonomous system. Some efforts have been made to solve this issue [15], [16], [17], [18]; most of them are now expired work and the rest have to be considered, at this moment, as work in progress.

In Fig. 5, we can see an example of interdomain system where the cooperation between PCEs is needed in order to compute a complete LSP from the ingress LER (in AS1) to egress LER (in AS2). Each PCE has the capability of compute path segments only over its own AS. In this situation, the PCE belonging to AS1 has to be able to discover dynamically the existence of a PCE in AS2 that can help it in the task of computing a complete LSP for ingress LER in AS1.

At present, the only existing solution lies in reaching agreements between the implied autonomous systems and setting up, in a manual way, the relationships between PCEs. Nevertheless, it is desirable that this tasks can be achieved in an automatically and dynamically way.

In this work, we contributes with a mechanism that covers this empty gap, still unsolved, allowing a PCC to discover the existence of other PCEs in an adjacent autonomous system. For that, we extend the discovery mechanisms proposed by IETF for the intra-AS environment.

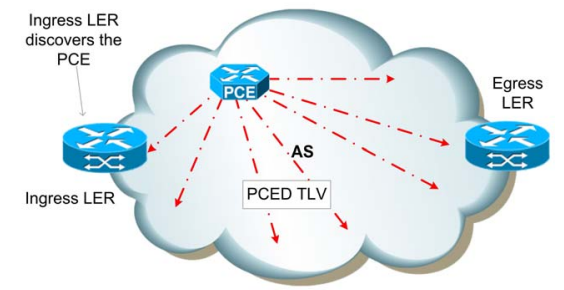

Fig. 4. PCED TLV used to discover PCEs via OSPF.

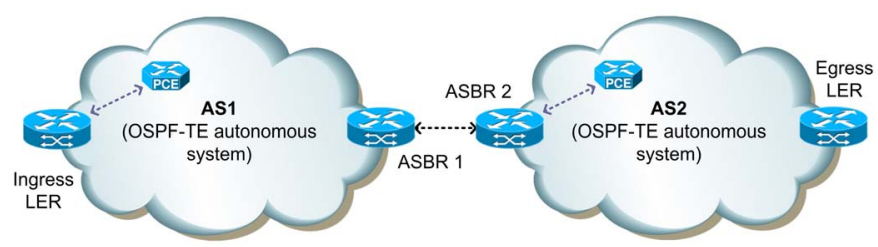

Fig. 5. Environment needing an inter-AS PCE discovery mechanism 


\section{PROPOSAL OF INTER-AS PCES DISCOVERY MECHANISM}

The discovery process inside an AS is, in its origin, different from interdomain discovery process. In the former, the goal is to make visible a PCE to those interested PCCs inside an AS, no-matter the way in which this process is carried out, whereas in the latter, a PCE has to be visible for other PCEs (that will act as PCCs) in a neighbor AS. It implies that the process must be careful with the privacy of the autonomous systems, avoiding undesirable disclosures.

Our proposal, PILEP, extends [19] by adding new features to allow a set of PCE in foreign autonomous systems to discover the existence of PCEs in the local AS. To achieve this objective, we have designed extensions to OSPF-TE and BGP (Border Gateway Protocol) since now, both are going to be the protocols that support the interdomain PCEs discovery mechanism.

\section{A. OSPF-TE extension: SET-VISIBLE-TO sub-TLV}

The first thing we need to make our proposal operative is to extend OSPF-TE. It has to preserve the capacity of spreading PCEs inside an AS but, in addition, it must be able to advertise to ASBRs (Autonomous System Border Router) the necessity of passing out the announcement over the AS boundary, from the local AS to an adjacent AS. To achieve this goal, we have designed an extension (Fig. 6) of PCED TLV used in the abovementioned interior discovery mechanism: a new subTLV called SET-VISIBLE-TO. This new sub-TLV is analogous to PCED but addressed to adjacent autonomous systems instead of the local AS.

SET-VISIBLE-TO is optional and has a variable size. It is located within PCED TLV and there can be more than an instance of the same per PCED. That allows counting on with differentiated sets of information to be discovered by each adjacent autonomous system. This operational mode allows PCEs in adjacent ASes to known about the existence of the advertised PCEs. They will discover that PCEs in different degrees and this fact enables network managers to establish more than one discovery policy depending on the target AS.

SET-VISIBLE-TO format (Fig. 7) is similar to PCED format. If a SET-VISIBLE-TO sub-TLV is present inside a PCED TLV, it must include at least four mandatory sub-subTLVs: SVT-PCE-ADDRESS, whose meaning is the same that PCE-ADDRESS in PCED; SVT-PATH-SCOPE, similar to

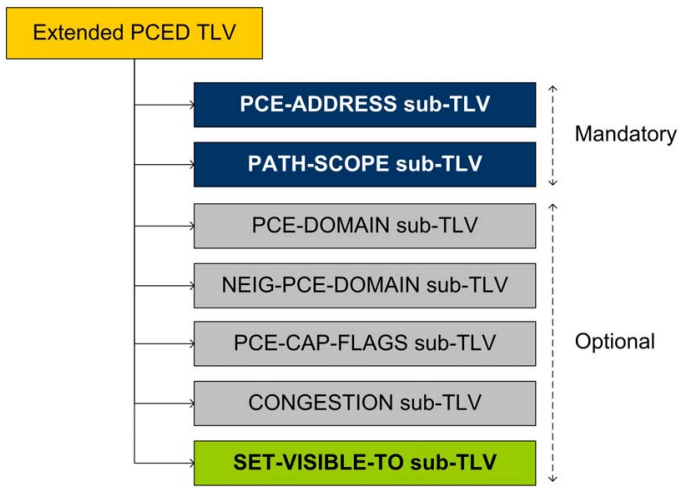

Fig. 6. Location of SET-VISIBLE-TO inside PCED.

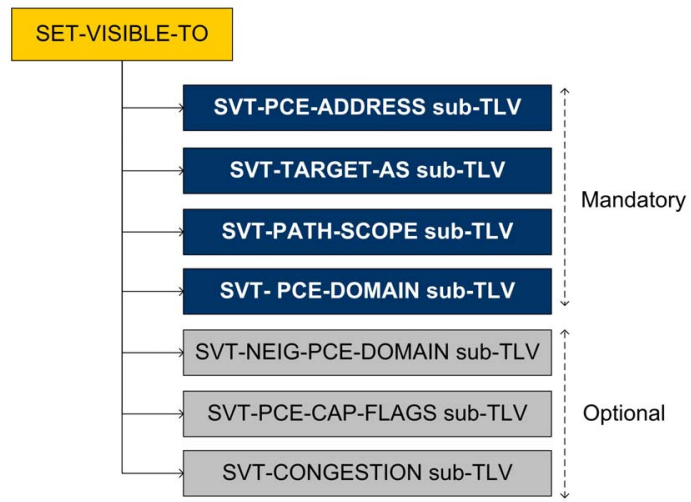

Fig. 7. Structure of SET-VISIBLE-TO sub-TLV.

PATH-SCOPE in PCED; SVT-PCE-DOMAIN, used to show the number of the AS that owns the advertised PCE; and SVTTARGET-AS (Fig. 8), which is a set of AS numbers where the SET-VISIBLE-TO has to be forwarded. Besides the mandatory sub-TLV, SET-VISIBLE-TO may contain other optional sub-TLVs intended to make easy the PCE selection process: SVT-NEIG-DOMAIN, which is a set of adjacent autonomous systems where the advertised PCE is able to computer LSP; lastly, SVT-PCE-CAP-FLAGS and SVTCONGESTION, whose meanings are the same that PCECAP-FLAGS and CONGESTION in PCED TLV, correspondingly. We can see a summarized view of SETVISIBLE-TO format in Table 1.

Using SET-VISIBLE-TO, a BGP router that at the same time takes part in OSPF-TE is able to understand that a PCED TLV has to traverse the border of the local AS and be forwarded to one or more adjacent autonomous systems. Likewise, it will know what information must show to each one of those adjacent ASes, as required in [14] for any wouldbe interdomain PCE discovery mechanism.

\section{B. BGP extension: $A S$ PCE attribute}

In the previous paragraph, we have designed a mechanism to make an ASBR know that it has to move the PCEs advertisements from the local AS to some other adjacent ASes. Next step is to endow BGP with the capacity of making that transfer real.

\section{SVT-TARGET-AS}

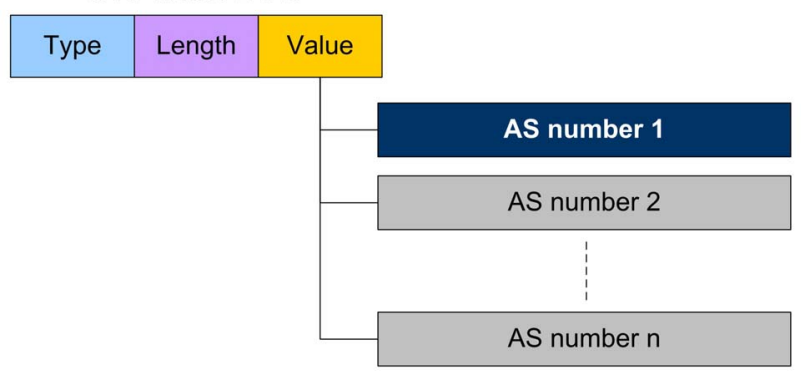

Type: 1 octet. Sub-sub-TLV type. To be defined by IANA. Length: 1 octet. Number of items in the 'Value' field.

Value: Sequences of AS numbers. Its size, in octets, is $2 x$ Length.

Fig. 8. Format of SVT-TARGET-AS. 
TABLE 1

SUMMARY OF SET-VISIBLE-TO SUB-TLV FORMAT

\begin{tabular}{|c|c|c|}
\hline Sub-sub-TLV & Mand. & Description \\
\hline SVT-PCE-ADDRESS & $\checkmark$ & IP address of the advertised PCE. \\
\hline SVT-TARGET-AS & $\checkmark$ & $\begin{array}{l}\text { Set of AS numbers of those ASes } \\
\text { that must know about the existence } \\
\text { of the advertised PCE. }\end{array}$ \\
\hline SVT-PATH-SCOPE & $\checkmark$ & $\begin{array}{l}\text { Identical values and meaning to } \\
\text { PATH-SCOPE, specified in [19]. }\end{array}$ \\
\hline SVT-PCE-DOMAIN & $\checkmark$ & $\begin{array}{l}\text { AS number of the AS that owns the } \\
\text { advertised PCE. As defined for } \\
\text { PCE-DOMAIN in [19], but } \\
\text { expressing only AS numbers. } \\
\text { Identical values to NEIG-PCE- }\end{array}$ \\
\hline SVT-NEIG-PCE-DOMAIN & $x$ & $\begin{array}{l}\text { DOMAIN defined in [19], but } \\
\text { expressing only AS numbers. }\end{array}$ \\
\hline SVT-PCE-CAP-FLAGS & $x$ & $\begin{array}{l}\text { Format and meaning identical to } \\
\text { PCE-CAP-FLAGS defined in [19]. }\end{array}$ \\
\hline SVT-CONGESTION & $x$ & $\begin{array}{l}\text { Format and meaning identical to } \\
\text { CONGESTION defined in [19]. }\end{array}$ \\
\hline
\end{tabular}

This is the second extension that we need to build an interdomain PCEs discovery mechanism based on OSPF-TE and BGP.

One remarkable BGP feature is the use of route attributes [23], to provide supplementary information for a given BGP route. In addition, it is defined how to make use of optional route attributes. This kind of attributes is important because not every BGP implementation has the obligation of recognizing it. In PILEP, we take advantage of this BGP feature to make a PCE advertisement reach an adjacent autonomous system.

We propose a new optional, non-transitive, BGP route attribute called AS_PCE (Fig. 9). Because of the fact that it is an optional and non-transitive attribute, it is not compulsory for an ASBR to understand it, and that new BGP attribute will not go beyond the target autonomous system. The content of AS_PCE is the same that the content of SET-VISIBLE-TO sub-TLV except for the field SVT-TARGET-AS belonging to SET-VISIBLE-TO, which is not included in AS PCE.

A BGP/OSPF-TE node that implements PILEP, will operate in the following way: if it detects, via OSPF-TE, a PCED TLV including at least a SET-VISIBLE-TO sub-TLV, it will analyze whether it share peering agreements with any of the ASes specified in SVT-TARGET-AS field or not. For each one of the ASes specified in SVT-TARGET-AS with which it share peering agreements, it will build an AS_PCE attribute

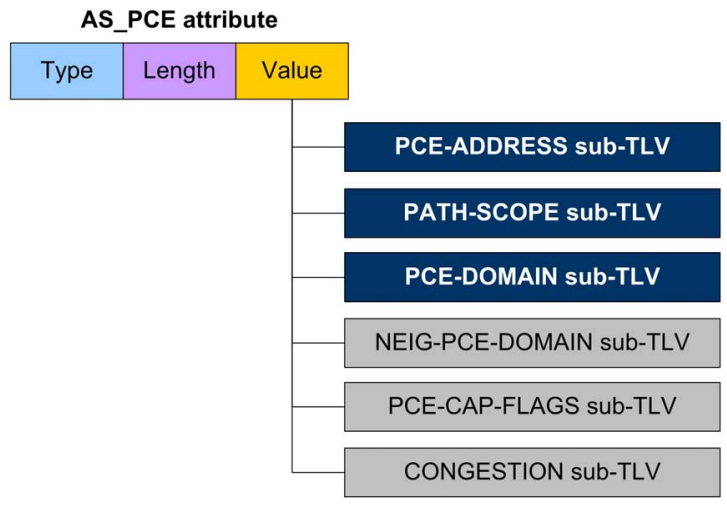

Fig. 9. Structure of AS_PCE route attribute and will add it in every route to the local AS that are waiting for being advertised in the BGP routing table (Adj-RIB-Out) [23]. If there are not routes to the local AS in the BGP routing table, the BGP node must generate one (NLRI should contain networks 'controlled' by the advertised PCE) and include the AS_PCE attribute within it. In this way, we accomplish that PCE advertisements cross the local AS boundary by way of using BGP UPDATE messages.

\section{UPDATE messages management in receiving ASBR}

BGP nodes perform a route selection process to choose the adequate routes for them. For that reason, they could reject the route that incorporates the AS_PCE attribute. In order to pick up that attribute before that potential rejection, the UPDATE messages management procedure should be as one can see in Fig 10. When a BGP/OSPF-TE node that implements PILEP receives a route, that node must check whether it incorporates a PCE announcement (an AS PCE attribute). If so, the BGP node must detour a copy of that route to the extended OSPFTE modules, which will process it. This is the proper module to succeed in doing this task because it is which has the capability of spreading routing information within the interior of the autonomous system.

Moreover, the BGP module will process the previously mentioned route, in a parallel way, as usual.

For its part, and beyond the selection process carried out by BGP, the extended OSPF-TE module will transform the AS_PCE attribute into a traditional PCED TLV in order to be spread jointly with RI-LSA messages inside the target autonomous system. This is a direct transformation because "Value" field in AS_PCE has an identical format than "Value" field in PCED TLV. From the beginning to the end, the PCED TLV will have gone through some changes: from an extended PCED containing a SET-VISIBLE-TO sub-TLV, to an AS_PCE attribute inside a BGP UPDATE message and, lastly, a traditional PCED TLV in the end autonomous system.

\section{EXPLANATORY EXAMPLE}

There are messages of different technologies involved in the suggested procedure. Therefore, to facilitate the understanding of PILEP global operation, we will apply each stage over the example showed in Fig. 5: a basic interdomain system containing two PCEs that need to cooperate.

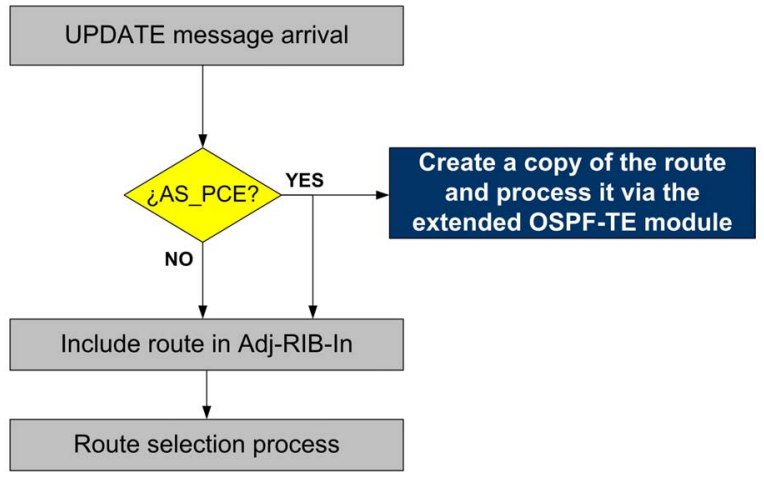

Fig. 10. Management of UPDATE messages containing AS_PCE 


\section{A. Spreading of $A S 2$ 's $P C E$ inside its $A S$}

The first step that AS2's PCE must follow is to spread itself inside its own AS. To do that, it uses the extended PCED TLV and the intradomain discovery mechanism defined by the IETF. PCED incorporates, in that case, a SET-VISIBLE-TO sub-TLV where some information is specified: its IP address; the AS number that identifies AS1; the capability of collaborating in interdomain LSP computation; and the capability of computing paths over AS2. Although it could include supplementary information, in this example we will suppose that there is not more information available. The extended PCED will be attached to the next OSPF-TE link state advertisement and will be spread inside AS2 (Fig. 11).

\section{B. PCE detection and inter-AS announcement transfer}

ASBR2 is a node that takes part in both BGP and OSPF-TE inside AS2. Furthermore, it implements PILEP. Therefore, when it receives the RI-LSA message (and the extended PCED that it includes), will discover a PCE inside its own AS; at the same time, due to the existence of the SET-VISIBLETO sub-TLV, it will understand that it must make that advertisement to cross AS2 edge. It is an ASBR, so it will process SET-VISIBLE-TO. By doing this, it detects that the announcement has to reach AS1, which ASBR 2 shares peering agreements with. Then, it builds an AS PCE attribute from the information contained in SET-VISIBLE-TO and includes it in every route to the local AS that is waiting for being advertised via BGP. The next time that ASBR 2 announces one of these routes to AS1, AS_PCE will go from AS2 to AS1 at the same time (Fig. 12).

\section{UPDATE message management in ASBR 1}

ASBR 1, as ASBR 2, takes part in both OSPF-TE and BGP inside AS1, and implements our proposal. It receives an UPDATE message from ASBR 2 and then it analyzes its attributes. It recognizes AS_PCE and next detours a copy of it to its extended OSPF-TE module to be treated. Concurrently, the route is stored in Adj-RIB-in (raw BGP routing table) until the route selection procedure takes place.

\section{Spreading of inter-AS PCE inside AS1}

OSPF-TE module belonging to ASBR 1 knows that must transform AS PCE into a usual PCED TLV and attach it to link state advertisements in order to spread the existence of AS2's PCE inside AS 1 (Fig. 13). Because of that spreading, AS1's PCE will discover the existence of AS2's PCE. In addition, it will realize that the external PCE belongs to AS2, can help it in an interdomain LSP computation, is able to compute paths over AS2 and, what is most important, its IP address is known. Therefore, AS1's PCE is capable of establishing a PCEP session to AS2's PCE (Fig. 14).

AS1's PCE will settle on one of the available PCEs to cooperate to compute the route that ingress LER has asked for. In this example, there are not more candidates and the chosen element is, therefore, AS2's PCE. After the whole process, the discovery mechanism has finished and the usual PCE operation mode will start.

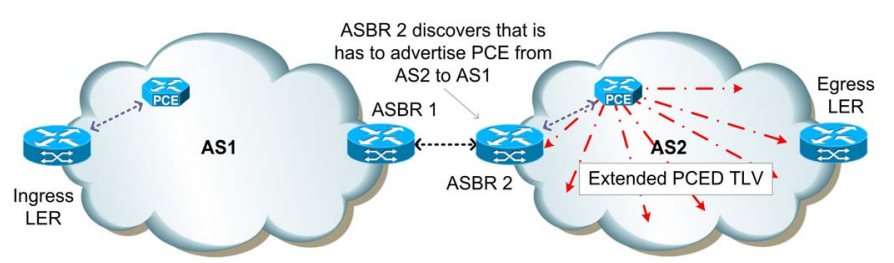

Fig. 11. Use of extended PCED TLV inside AS2.

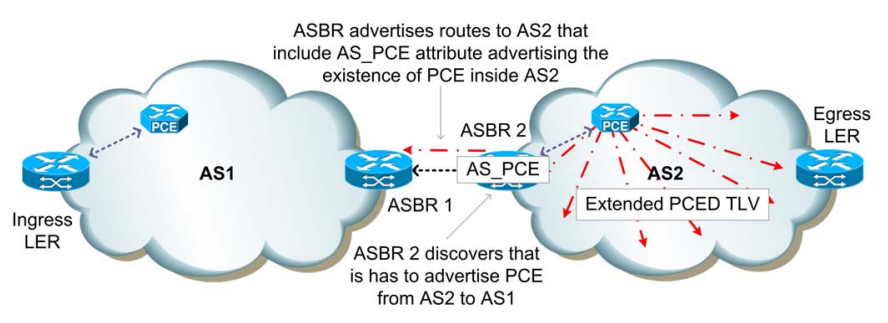

Fig. 12. Using AS_PCE in an UPDATE message.

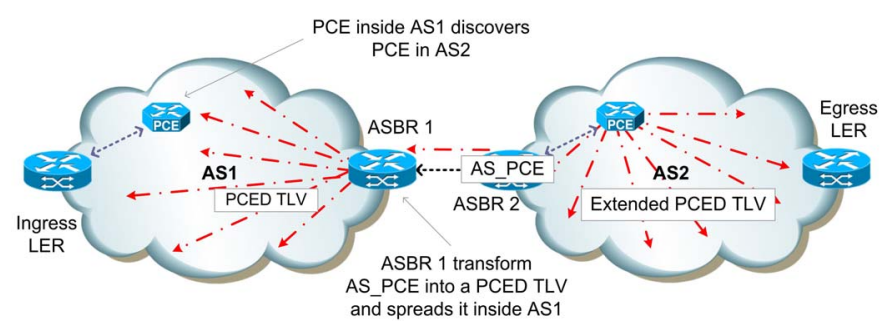

Fig. 13. Effect of transforming AS_PCE into TLV PCED.

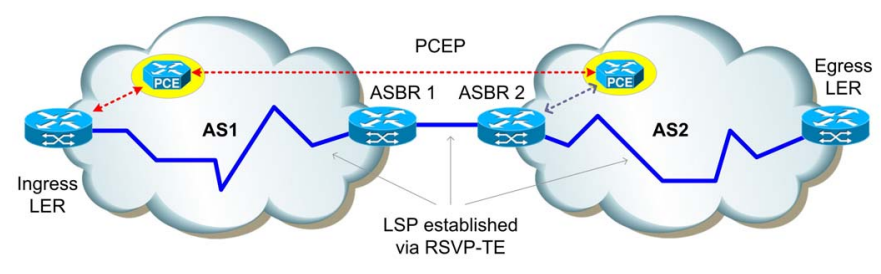

Fig. 14. PCE-based inter-AS LSP computation

\section{CONCLUSIONS AND FUTURE WORK}

In this research work, still in progress, we propose PILEP, a mechanism to allow the dynamical discovery of PCEs in an interdomain environment. This method is justified due to the necessity of cooperation between PCEs involved in interdomain LSP computation and, of course, because there is not an alternate interdomain PCE discovery technique.

Our proposal follows the work started by the IETF when using the mechanisms existing in the traditional routing protocols to make the discovery process easier. We have designed a new optional sub-TLV, SET-VISIBLE-TO, that could be incorporated inside an OSPF PCED TLV and a new BGP attribute, optional and non-transitive, AS_PCE, to be included in the routes advertised by BGP via UPDATE messages.

We can summarize the advantages of PILEP as follows:

- It covers the necessity of having a mechanism to discover PCEs in interdomain environments.

- It allows the establishment of diverse discovery policies, depending on the target AS. 
- To make its work, it needs minimum changes in the concerned protocols.

Since this is an ongoing work, we still have to perform some tasks in the near future; for example, we have to assess and analyze the goodness of the proposed mechanism using simulations (we are at this moment evaluating some network simulators), or we need to extend PILEP to make it work in combination to IS-IS.

\section{ACKNOWLEDGEMENTS}

This research work is sponsored in part by the regional Education, Science and Technology Ministry belonging to the Extremadura (Spain) Regional Government, through the AGILA-2 project numbered as PR1A06145; and by the national Industry, Tourism and Commerce Ministry belonging to Spain National Government, through MESEAS project numbered as FIT-350301-2007-14.

\section{REFERENCES}

[1] D. Awduche, A. Chiu, A. Elwalid, I. Widjaja, X. Xiao. Overview and Principles of Internet Traffic Engineering. IETF RFC 3272. May, 2002.

[2] E. Crawley, R. Nair, B. Rajagopalan, H. Sandick. A Framework for QoS-based Routing in the Internet. IETF RFC 2386. August, 1998.

[3] E. Rosen, A. Viswanathan, R. Callon. Multiprotocol Label Switching Architecture. IETF RFC 3031. January, 2001.

[4] C. K. Chau, R. Gibbens, T. G. Griffin. Towards a Unified Theory of Policy-Based Routing. INFOCOM 2006, pp. 1-12. April, 2006.

[5] A. Sprintson, M. Yannuzzi, A. Orda, X. Masip-Bruin. Reliable Routing with QoS Guarantees for Multi-Domain IP/MPLS Networks. INFOCOM 2007, pp. 1820-1828. May, 2007.

[6] A. Farrel, J. P. Vasseur, J. Ash. A Path Computation Element (PCE)Based Architecture. IETF RFC 4655. August, 2006.

[7] J. Ash, J.L. Le Roux. Path Computation Element (PCE) Communication Protocol Generic Requirements. IETF RFC 4657. September, 2006.

[8] J. P. Vasseur, J. L. Le Roux. Path Computation Element (PCE) communication Protocol (PCEP). IETF Draft draft-ietf-pce-pcep-09.txt. Work in progress. November, 2007.

[9] D. Awduche, L. Berger, D. Gan, T. Li, V. Srinivasan, G. Swallow. RSVP-TE: Extensions to RSVP for LSP Tunnels. IETF RFC 3209. December 2001.

[10] M. Yannuzzi, X. Masip-Bruin, O. Bonaventure. Open issues in interdomain routing: a survey. IEEE Network. Volume 9, Issue 6. November-December, 2005.

[11] C. Pelsser, S. Uhlig, O. Bonaventure. On the difficulty of establishing interdomain LSPs. Proceedings of the IEEE Workshop on IP Operations and Management. October 2004.

[12] R. Zhang, J.P. Vasseur. MPLS Inter-Autonomous System (AS) Traffic Engineering (TE) Requirements. IETF RFC 4216. November, 2005.
[13] J.L. Le Roux, J.P. Vasseur, J. Boyle. Requirements for Inter-Area MPLS Traffic Engineering. IETF RFC 4105. June, 2005.

[14] J.L. Le Roux. Requirements for Path Computation Element (PCE) Discovery. IETF RFC 4674. October, 2006.

[15] K. Kumaki, T. Murai. BGP protocol extensions for Path Computation Element (PCE) Discovery in a BGP/MPLS IP-VPN. IETF Draft draftkumaki-pce-bgp-disco-attribute-01.txt. April, 2008. Work in progress.

[16] Vijayanand, Somen Bhattacharya, Prasanna Kumar. BGP protocol extensions for PCE Discovery across Autonomous Systems. IETF Draft draft-vijay-somen-pce-disco-proto-bgp-04.txt. July, 2007. Expired I-D.

[17] M. Boucadair, P. Morand. Path Computation Service discovery via Border Gateway Protocol. May, 2005. IETF Draft draft-boucadair-pcediscovery-01.txt. Expired I-D.

[18] M. Boucadair, P. Morand. A Solution for providing inter-AS MPLSbased QoS tunnels. IETF Draft draft-boucadair-pce-interas-01.txt. May 2005. Expired I-D.

[19] J. L. Le Roux, JP. Vasseur, Y. Ikejiri, R. Zhang. OSPF Protocol Extensions for Path Computation Element (PCE) Discovery. IETF RFC 5088. January 2008.

[20] J. L. Le Roux, JP. Vasseur, Y. Ikejiri, R. Zhang. IS-IS Protocol Extensions for Path Computation Element (PCE) Discovery. IETF RFC 5089. January 2008.

[21] A. Lindem, N. Shen, J. P. Vasseur, R. Aggarwal, S. Shaffer. Extensions to OSPF for Advertising Optional Router Capabilities. IETF RFC 4970. July, 2007.

[22] D. Katz, K. Kompella, D. Yeung. Traffic Engineering (TE) Extensions to OSPF Version 2. IETF RFC 3630. September, 2003.

[23] Y. Rekhter, T. Li, S. Hares. A Border Gateway Protocol 4 (BGP-4). IETF RFC 4271. January, 2006.

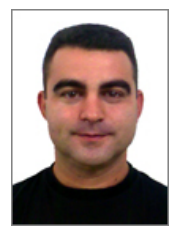

M. Domínguez-Dorado, Zafra (Badajoz). SPAIN, 1977. He obtained his Master in Advanced Studies, his B.Sc. in Computer Science and his M.Sc. in Computer Science at The University of Extremadura (UEx) in 2007, 2006 y 2004, correspondingly. He worked for some companies related to Computer Science and came back to UEx, where he is now researching into PCE, MPLS-TE and QoR.

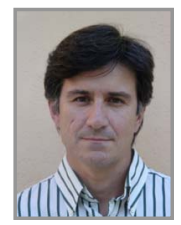

José-Luis González-Sánchez, obtained his Ph.D. at Technical School of Catalunya. Now he is a full-time assistant professor and coordinates the Telematics Engineering Area of DISIT department at The University of Extremadura. He is the main researcher of GITACA research group. His areas of interest are Quality of Service (QoS), MPLS-TE and the security in communications.

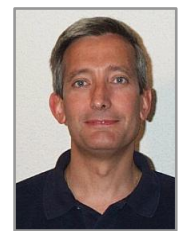

J. Domingo-Pascual, is a full professor at DAC department of Technical School of Catalunya (UPC). He is co-founder and researcher of the Broadband Advanced Communications Center. His areas of interest are broadband communications and their applications, IP/ATM integration, QoS provision and management, traffic engineering, IP traffic analysis and characterization. 